\title{
Institutionelle Regeln oder politische Kultur? Faktoren der parlamentarischen Mitgliederfluktuation in der Schweiz
}

\author{
Antoinette Feh Widmer und Adrian Vatter ${ }^{*}$
}

\section{Einleitung}

Der personelle Wechsel in Parlamenten ist ein wichtiger Bestandteil der Demokratie. Ihr Funktionieren setzt dabei ein Gleichgewicht zwischen einem umfassenden Wechsel und vollständiger Stabilität des parlamentarischen Personals voraus. Entzieht man dem Bürger die Möglichkeit, gewählte Repräsentanten nach Ablauf einer Legislaturperiode abzuwählen, wird nicht nur das Prinzip der Responsivität der Parlamentarier gegenüber den Wählern verletzt, sondern es droht auch die Gefahr einer Tyrannei ${ }^{1}$. Auch schon bei einer ausgeprägten Stagnation des Mitgliederwechsels lässt sich argumentieren, dass der mit der fehlenden personellen Veränderung einhergehende Ideenstillstand in einem Parlament dazu führen kann, dass ein Politikwandel im Sinne der gewandelten Bevölkerungspräferenzen zunehmend unwahrscheinlicher wird. Der Vorteil der Fluktuation von politischen Repräsentanten liegt dabei auf der Hand: Mit neuen Köpfen kommen neue Ideen in die Politik. Zu hoch sollte allerdings die Wechselrate nicht sein, sonst droht ein Verlust an Fachwissen und wichtigen politischen Erfahrungen. Zudem wirkt sich eine zu hohe parlamentarische Fluktuation negativ auf die Wirksamkeit und Effektivität eines Parlaments aus. Es stellt sich deshalb die Frage nach einer „optimalen“ parlamentarischen Fluktuation und ihren Ursachen. In der Forschung herrscht ein Konsens darüber, dass ein Mitgliederwechsel auf der einen Seite die Handlungsfähigkeit von Parlamenten erhalten soll und auf der anderen Seite aber den wandelnden gesellschaftlichen Interessenlagen gerecht werden muss ${ }^{2}$. Best und Cotta $^{3}$ sprechen in ihrer Analyse europäischer Mandatsträger von einem optimalen Mitgliederwechsel, welcher sich in der Regel im Wertebereich zwischen 20 und 30\% bewegen sollte.

Der vorliegende Beitrag liefert für die Schweiz eine umfassende Analyse der parlamentarischen Mitgliederfluktuation auf subnationaler Ebene für alle 26 kantonalen Legislativen für den Zeitraum zwischen 1960 und 2012. Im Mittelpunkt steht dabei die Frage, ob es eher institutionelle Regeln oder Merkmale der politischen Kultur sind, die den personellen Wechsel in Parlamenten erklären. Während bisherige Studien das Augenmerk hauptsächlich auf den Einfluss von politisch-institutionellen Elementen des Wahl- und Parlamentssystems auf den parlamentarischen Mitgliederwechsel gelegt

\footnotetext{
* Dieser Beitrag ist im Rahmen des vom Schweizerischen Nationalfonds finanzierten Forschungsprojekts „Parlamentarische Mitgliederfluktuation in der Schweiz“ (Projekt-Nr. 100017E_133395) entstanden.

${ }^{1}$ Vgl. Philip Manow, Electoral Rules and Legislative Turnover: Evidence from Germany’s Mixed Electoral System, in: West European Politics, 30. Jg. (2007), H. 1, S. 195 - 207.

${ }^{2}$ Vgl. Till Heinsohn / Markus Freitag, Institutional Foundations of Legislative Turnover: A Comparative Analysis of the Swiss Cantons, in: Swiss Political Science Review 18. Jg. (2012), H. 3, S. 352 - 370.

${ }^{3}$ Vgl. Heinrich Best / Maurizio Cotta, Parliamentary Representatives in Europe, 1848-2000: Legislative Recruitment and Careers in Eleven European Countries, Oxford 2000, S. 505.
} 
haben, interessiert sich der vorliegende Artikel auch für die Rolle politisch-kultureller Einflüsse, die in den bisherigen Studien zum Thema nur am Rande berücksichtigt wurden. Es sind aufgrund des Forschungsinteresses gleich verschiedene helvetische Besonderheiten, ${ }^{4}$ die dafür sorgen, dass der Fall Schweiz eine ideale Untersuchungsanlage für eine Analyse parlamentarischer Fluktuation bildet. Dazu zählt erstens, dass sich die Schweiz mit ihren weltweit mit am stärksten ausgebauten föderalen Strukturen besonders für einen subnationalen Vergleich eignet. Die Kantone als quasi-souveräne politische Gebilde mit eigenen Verfassungen, Parlamentsstrukturen und zahlreichen Kompetenzen bieten sich in ausgezeichneter Weise für eine komparative Betrachtung an. So erlauben sie einen Vergleich von eigenständigen Systemen, die einerseits über eine hohe Varianz des zu untersuchenden Phänomens verfügen, gleichzeitig aber über eine gemeinsame Geschichte und übergeordnete bundestaatliche Strukturen, wodurch zahlreiche - und für die Untersuchung zweitrangige - Merkmale stabil gehalten werden können. Mit anderen Worten: sie erfüllen die Voraussetzungen für einen mostsimilar case Vergleich in optimaler Weise. Zweitens eint die Schweiz im Gegensatz zu den meisten Nationalstaaten Europas nicht eine gemeinsame Sprache, Konfession oder Kultur, sondern ihre gemeinsame Institutionenarchitektur. Als politische Willensnation auf multikultureller Grundlage, die sich neben ihrer Mehrsprachigkeit durch einen starken Föderalismus und ausgebaute Mitwirkungsrechte auszeichnet, bietet sie eine einmalige Untersuchungsanlage, um den unterschiedlichen Einfluss von institutionellen Regeln und kulturellen Eigenheiten gleichzeitig zu untersuchen. Drittens erlaubt die Besonderheit des sog. Milizparlamentarismus in der Schweiz, also die Ausübung des parlamentarischen Mandats ausschliesslich durch Nicht-Berufspolitiker, die Wirkungsanalyse dieser auf den verschiedenen Staatsebenen praktizierten Form der nebenamtlichen Ausübung der politischen Repräsentation auf den politischen Wechsel in der Legislative. Viertens besteht eine Forschungslücke hinsichtlich empirisch-quantitativer Studien zu den Determinanten der Parlamentsfluktuation in der Schweiz. ${ }^{5}$

Der Beitrag ist wie folgt aufgebaut: nach einem Überblick über die parlamentarische Mitgliederfluktuation in den Schweizer Kantonen werden mögliche Erklärungsfaktoren für den parlamentarischen Mitgliederwechsel auf kantonaler Ebene begründet, wobei der Schwerpunkt bei politisch-institutionellen und politisch-kulturellen Determinanten liegt. Danach werden die empirischen Befunde in verschiedenen Einzelmodellen und danach in einem Gesamtmodell präsentiert. Folgerungen runden den Beitrag ab.

\footnotetext{
${ }^{4}$ Adrian Vatter, Das politische System der Schweiz, Baden-Baden 2014.

${ }^{5}$ Ausnahmen bilden die Studien auf kommunaler Ebene von Bettina Nyffeler / Annina Jegher / Stefan Spycher, Analyse der Rücktritte aus dem Berner Stadtrat. Büro für arbeits- und sozialpolitische Studien Bern (BASS) 1995 und Isabel Käslin / Raphael Anklin / Madleina Balmer / Tobias Graber, Rücktritte im Grossen Rat BaselStadt. Universität Basel 2009 sowie auf kantonaler Ebene von Till Heinsohn / Markus Freitag, a.a.O. (Fn. 2).
} 
2. Parlamentarischer Mitgliederwechsel in den Kantonen - Konzeptualisierung und Grundlagen

Im Vergleich zum schweizerischen Bundesparlament verfügen die kantonalen Parlamente, deren Grösse zwischen 49 und 180 Sitzen schwankt, aufgrund von zwei institutionellen Besonderheiten über eine relativ schwache Stellung gegenüber den anderen politischen Akteuren. So werden einerseits die gesetzgeberischen Kompetenzen der kantonalen Legislativen durch die ausgebauten direktdemokratischen Instrumente beschnitten, andererseits wird die Exekutive in den Kantonen wie in präsidentiellen Systemen direkt von der Bevölkerung gewählt. Damit ist die kantonale Regierung vom Parlament unabhängig, weshalb „klassische“ Regierungskrisen nicht eintreten können. Gleichzeitig kann die kantonale Legislative nicht über die Auflösung der Regierung entscheiden und Neuwahlen ausschreiben. Zusammenfassend sind die Volkswahl der Regierung, das obligatorische Gesetzesreferendum und die grosse Offenheit der Volksinitiative sowie die geringen Professionalisierungsstrukturen (d.h. keine Berufsparlamentarier) die wichtigsten Faktoren, welche die Stellung der kantonalen Parlamente relativieren und ihre Oberaufsichts- und Kontrollfunktionen in den Kantonen begrenzen. Die Wahlfunktion der kantonalen Parlamente beschränkt sich zudem auf die Bestellung des obersten kantonalen Gerichts und in einzelnen Kantonen auf die Wahl des Regierungspräsidenten.

Die in unserer Untersuchung verwendete Definition von Mitgliederwechsel orientiert sich im Wesentlichen an den Vorarbeiten von Rosenthal ${ }^{6}$, Matland und Studlar ${ }^{7}$ sowie Heinsohn und Freitag. Entsprechend wird parlamentarische Mitgliederfluktuation als der Anteil der Mitglieder eines kantonalen Parlaments definiert, die von einer Wahl zur andern ausgetauscht werden. Die weitaus häufigste Form für einen Wechsel ist der Rücktritt eines Parlamentariers - sei dies ordentlich (zum Ende einer Legislatur) oder ausserordentlich (während der Legislatur). Der Anteil abgewählter und nicht wieder nominierter Parlamentarier dagegen ist sehr gering.

\footnotetext{
${ }^{6}$ Vgl. Alan Rosenthal, Turnover in State Legislatures, in: American Journal of Political Science, 18. Jg. (1974), H. 3, S. $609-616$.

${ }^{7}$ Vgl. Richard E. Matland / Donely T. Studlar, Determinants of Legislative Turnover: A Cross-National Analysis, in: British Journal of Political Science, 34 Jg. (2004), H. 1, S. 87 - 108.

${ }^{8}$ Vgl. Till Heinsohn / Markus Freitag, a.a.O. (Fn. 2).
} 
Abbildung 1: Überblick über die kantonalen Gesamtmitgliederfluktuationsraten (1960-2012)

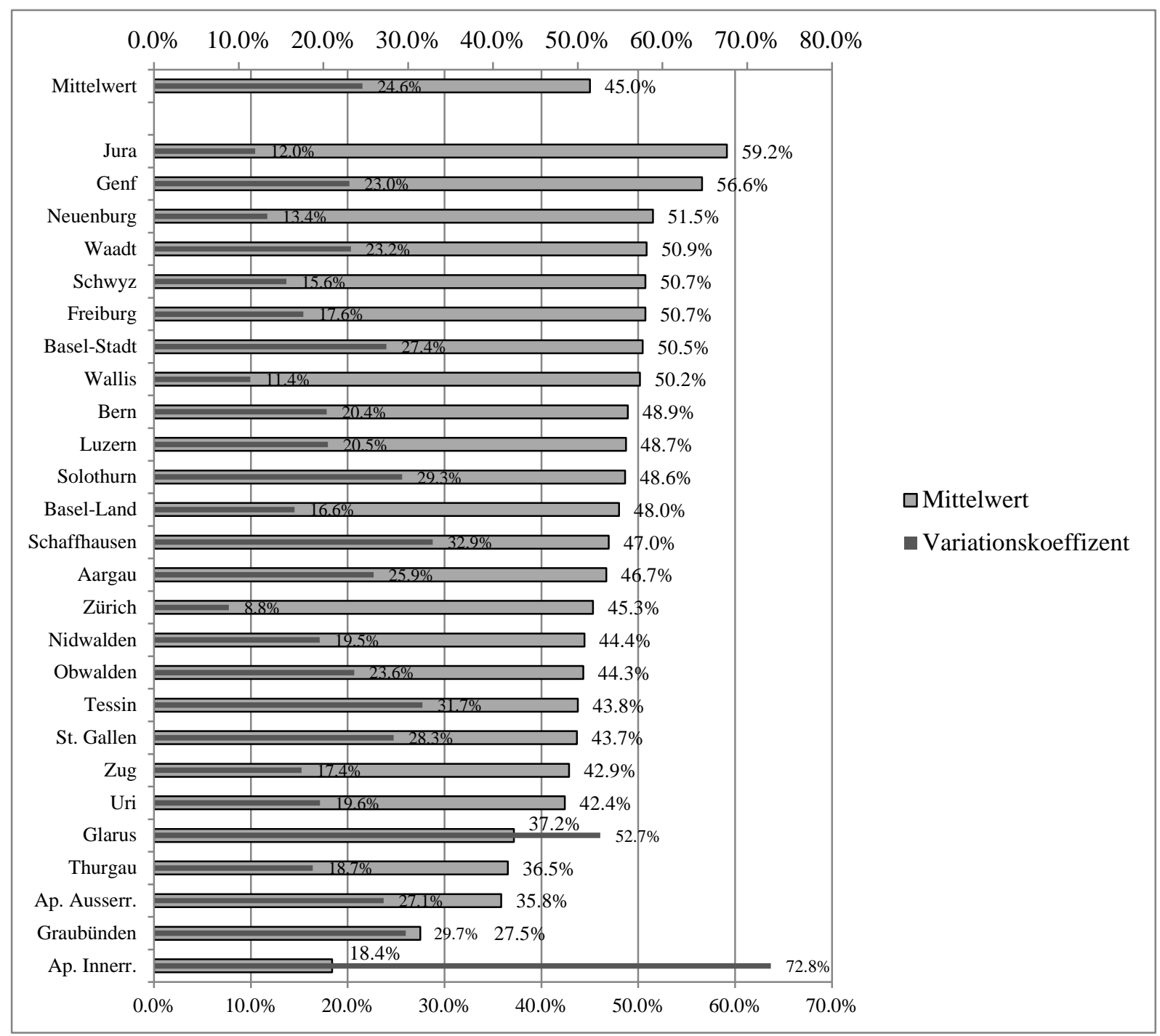

Abbildung 1 liefert einen ersten Überblick über die durchschnittlichen kantonalen Mitgliederfluktuationsraten zwischen 1960 und 2012. Dabei wird einerseits die beträchtliche Varianz hinsichtlich der parlamentarischen Mitgliederfluktuationsrate zwischen den Kantonen deutlich. So beträgt der Unterschied zwischen dem Kanton mit der kleinsten parlamentarischen Mitgliederfluktuationsrate, Appenzell Innerrhoden (18.4\%) und dem Kanton Jura mit dem grössten Anteil an parlamentarischem Mitgliederwechsel (59.2\%) 40.8 Prozentpunkte. Andererseits variiert die parlamentarische Mitgliederfluktuationsrate auch innerhalb der Kantone $\mathrm{zu}$ den einzelnen Beobachtungszeitpunkten beträchtlich, was anhand der Werte für den Varianzkoeffizienten ${ }^{9}$ ersichtlich wird. Die kantonale parlamentarische Gesamtmitgliederfluktuationsrate sowie die innerkantonale Varianz zwischen den Beobachtungszeitpunkten betragen über den Beobachtungszeitraum von 1960 bis 2012 einerseits 45.0\% sowie andererseits 24.6\% und sind in

\footnotetext{
${ }^{9}$ Varianzkoeffizient multipliziert mit 100. Der Variationskoeffizient steltt eine Normierung der Varianz dar: Ist die Standardabweichung größer als der Mittelwert, so ist der Variationskoeffizient größer 1. Die Aussagekraft liegt darin, dass eine Variable mit großem Mittelwert im Allgemeinen eine größere Varianz aufweist als eine mit einem kleinen Mittelwert.
} 
Abbildung 1 als Mittelwert ausgewiesen. Der Wert für die Gesamtmitgliederfluktuationsrate liegt damit deutlich über dem in der Literatur genannten „optimalen“ parlamentarischen Mitgliederwechsel von 20 bis 30 Prozent $^{10}$, was im Übrigen auch für die deutschen Bundesländer gilt. ${ }^{11}$ Nachfolgend werden nun mögliche Erklärungsfaktoren für die Varianz der Parlamentarierfluktuation hergeleitet.

\section{Erklärungsfaktoren für den parlamentarischen Mitgliederwechsel auf kantonaler Ebene}

\subsection{Politisch-institutionelle Einflussfaktoren}

Der Argumentation der neoinstitutionellen Ansätze folgend ${ }^{12}$, beeinflussen Institutionen und ihre Ausgestaltung die Entscheidungen politischer Akteure. Institutionelle Regeln setzen Anreize, Vorgaben und Schranken. Tatsächlich dokumentieren einige Studien, ${ }^{13}$ wie Institutionen und deren Ausgestaltung den parlamentarischen Mitgliederwechsel massgeblich bestimmen. ${ }^{14}$ Nachfolgend werden nun die möglichen Ursachen für die parlamentarische Fluktuation in Anlehnung an den neoinstitutionellen Theoriestrang formuliert.

\section{Modell 1: Wahlregeln}

Kantonale Verfassungen resp. die jeweiligen Wahlgesetze stellen den institutionellen Rahmen dar, in welchem sich die parlamentarischen Akteure bewegen und legen weitere Regeln fest, die einen Einfluss auf den parlamentarischen Mitgliederwechsel ausüben können, so insbesondere die Ausgestaltung der Amtszeitbeschränkung.

\section{Wahlsystem}

Das Wahlsystem mit seinen unterschiedlichen Ausprägungen wird in Studien immer wieder als signifikante Einflussgrösse auf den parlamentarischen Mitgliederwechsel bezeichnet ${ }^{15}$. Proporz- resp. Majorzsysteme gewichten die persönlichen Eigenschaften der Kandidierenden unterschiedlich. Majorzwahlen ermöglichen es bisherigen Mandatsträgern gegenüber Herausforderern anderer Parteien sich zu profilieren, indem sie sich auf ihre Reputation und ihren Leistungsausweis beziehen können. Dieser sogenannte Amtsinhaber-Bonus kann bei

\footnotetext{
${ }^{10}$ Vgl. Heinrich Best / Maurizio Cotta, a.a.O. (Fn. 3).

${ }^{11}$ Gemäss Till Heinsohn, Mitgliederfluktuation in den Parlamenten der deutschen Bundesländer, LIT Verlag, Münster 2014, S. 235 liegt die Mitgliederfluktuationsrate bei über 50 \%.

${ }^{12}$ Vgl. James G. March / Johan P. Olson, Rediscovering Institutions, New York 2010.

${ }^{13}$ Vgl. Kenneth Green, An Economic Investigation of Interstate Variation in Legislative Turnover, in: Public Finance Quarterly, 21. Jg. (1993), H. 1, S. 84 - 99.; Wayne L. Francis / Lawrence Kenny, Equilibrium Projections for the Consequences of Term Limits upon Expected Tenure, Institutional Turnout, and Membership Experience, in: Journal of Politics 59. Jg. (1997), S. 240 - 252.

${ }^{14}$ Vgl. Yanna Krupnikov / Rebecca B. Morton / Charles S. Shipan, Voter Uncertainty, Political Institutions, and Legislative Turnover, Draft 2008, S. 3 ff.

${ }^{15}$ Vgl. Till Heinsohn / Markus Freitag, a.a.O. (Fn. 2).; Till Melchert, Die Schweizer Kantonsparlamente im Vergleich - Eine Quantitativ-Statistische Analyse des Mitgliederwechsels, Diplomarbeit Universität Konstanz 2009.; Richard E. Matland / Donley T. Studlar, a.a.O. (Fn. 7).
} 
einer umstrittenen, knappen Wahl zu einer Situation führen, bei welcher der Gewinner im Gegensatz zum Verlierer alles bekommt ("winner takes it all"-Situation). Demgegenüber leistet das Proporzwahlsystem dem Amtsinhaber-Bonus weniger Vorschub, da gewonnene Parlamentsmandate proportional zum Wähleranteil unter den politischen Parteien verteilt werden. Wählerentscheide basieren bei Proporzwahlen eher auf parteipolitischen Aspekten als auf den individuellen Eigenschaften der Kandidierenden. Matland und Studlar ${ }^{16}$ stellen in ihrer Studie fest, dass das Proporzwahlsystem zu einer höheren parlamentarischen Fluktuationsrate führt als das Majorzwahlsystem. Dies deshalb, da politische Parteien bei einer Majorzwahl wohl kaum den Austausch von gestandenen Amtsträgern zulassen würden, wenn dieser Wechsel mit dem Risiko eines Sitzverlustes verbunden ist. Entsprechend ist davon auszugehen, dass mögliche Niveauunterschiede der parlamentarischen Fluktuationsraten mit den unterschiedlichen Wahlsystemen in den Kantonen erklärt werden können.

\section{Amtszeitbeschränkung}

Ebenfalls einen positiven Einfluss auf den parlamentarischen Mitgliederwechsel stellt Moncrief $^{17}$ durch das Vorhandensein einer Amtszeitbeschränkung bzw. durch deren Länge fest. Diese institutionelle Regel sorgt einerseits dafür, dass Mandatsträger nach einer bestimmten Amtsdauer die Legislative verlassen müssen und andererseits, dass eine erneute Kandidatur nach dem Ausscheiden verhindert wird. In den Kantonen Basel-Stadt und Jura sind die Parlamentarier etwa gezwungen, nach zwölf aufeinanderfolgenden Jahren ihr Mandat niederzulegen und auf eine erneute Kandidatur zu verzichten. Auch wenn es sich bei einer Amtszeitlimitierung von zwölf Jahren um eine grosszügige Vorschrift handelt, besteht die Annahme, dass eine solche institutionelle Beschränkung einen unmittelbaren Einfluss auf den parlamentarischen Mitgliederwechsel ausübt.

\section{Modell 2: parlamentarischer Handlungsrahmen}

Wie eingangs erläutert, strukturiert der institutionelle Rahmen die Handlungen der Akteure, indem er deren verfügbare Ressourcen benennt. Dabei wird angenommen, dass ein durch den institutionellen Rahmen stark beschränkter Handlungsspielraum für die am parlamentarischen Prozess beteiligten Akteure zu einem höheren Mitgliederwechsel führt, geringere

\footnotetext{
${ }^{16}$ Vgl. Richard E. Matland / Donley T. Studlar, a.a.O. (Fn. 7).

${ }^{17}$ Vgl. Gary F. Moncrief / Richard G. Niemi / Lynda W. Powell, Time, Term Limits, and Turnover: Trends in Membership Stability in U.S. State Legislatures, in: Legislative Studies Quarterly, 29. Jg. (2004), H. 3, S. 357 381.
} 
Handlungsmöglichkeiten eine optimale Durchsetzung der eigenen Präferenzen verhindert. In der vorliegenden Analyse wird die Ressourcenverfügbarkeit der Parlamentarier und damit der Handlungsspielraum der Legislative gegenüber der Exekutive durch drei Variablen erfasst: 1) die Möglichkeit der Legislative, eine parlamentarische Untersuchungskommission mit weitgehenden Sonderrechten einzusetzen, ${ }^{18}$ 2) die Anzahl der ständigen Kommissionen sowie 3) Vorhandensein eines ständigen Parlamentsdienstes. Entsprechend wird postuliert, dass mögliche Niveauunterschiede der kantonalen parlamentarischen Fluktuationsraten durch die unterschiedliche Ausgestaltung des legislativen Handlungsspielraums erklärt werden kann.

\section{Modell 3: politisch-institutionelle Stabilität}

Parlamentsverkleinerungen

Im Gegensatz zu anderen institutionellen Faktoren wurde der Einfluss von Parlamentsverkleinerungen auf den Mitgliederwechsel bis heute kaum untersucht, obwohl das Phänomen immer häufiger auftritt. In den letzten Jahren haben etwa in der Schweiz zehn Kantone ihre Legislativen um durchschnittlich einen Viertel verkleinert ${ }^{19}$. Parlamentsverkleinerungen können dabei aufgrund der Reduktion der bei einer Wahl zu vergebenden Sitze bei manchen Mandatsträgern am Ende einer Legislaturperiode zu einem freiwilligen Ausscheiden führen, weil sie das erhöhte Risiko einer Abwahl nicht eingehen möchten. Gleichzeitig kann angenommen werden, dass eine Parlamentsverkleinerung auch eine erhöhte Konkurrenzsituation zwischen erneut kandidierenden Mandatsträgern und aufstrebenden Kandidierenden erzeugt. Diese Konkurrenzsituation führt wiederum zu einem intensiver geführten Wahlkampf, der es aufstrebenden Kandidierenden besser ermöglicht, sich gegenüber bisherigen Parlamentarier zu profilieren und für ein Amt gewählt zu werden.

\section{Wählervolatilität}

Das Phänomen des retrospektiven Wählens zeigt, dass Wähler, welche mit den Leistungen ihrer Repräsentanten unzufrieden sind, diese oft in der folgenden Wahl abstrafen, d.h. nicht für eine weitere Amtsperiode bestätigen. Gleichzeitig werden bisherige Mandatsträger durch aufstrebende Jungpolitiker in ihren Ämtern ersetzt ${ }^{20}$. Dieser Argumentation folgend sind diese

\footnotetext{
${ }^{18}$ Gemäss der Untersuchung von Stepahnie Kaiss, Das Verhältnis zwischen Exekutive und Legislative in den Schweizer Kantonen. Das Ausmass der Exekutivdominanz auf kantonaler Ebene, Diplomarbeit Universität Zürich 2010, kann dieser Indikator als Annäherungsgrösse für die Stärke der kantonalen Legislative gegenüber der kantonalen Exekutive betrachtet werden.

${ }^{19}$ Vgl. Till Heinsohn / Markus Freitag, a.a.O. (Fn. 2), S. 359.

${ }^{20}$ Vgl. G. Bingham Powell Jr., The Chain of Responsiveness, in: Journal of Democracy, 15. Jg. (2004), H. 4, S. $91-105$.
} 
sogenannten Wechselwähler für die Wählervolatilität verantwortlich ${ }^{21}$. Matland und Studlar²2 stellen fest, dass die Wahrscheinlichkeit eines Amtsinhabers seinen Sitz an einen Herausforderer zu verlieren, von einer Wahl zur nächsten ansteigt, sofern dessen Partei schon zuvor stets Sitze dazu gewonnen bzw. verloren hat. Auch Rosenthal ${ }^{23}$ argumentiert, dass die absolute Sitzzahl einer Partei in einer Legislative stabil ist, wenn die Wahrscheinlichkeit eines Sitzverlustes dieser Partei geringer ist als für andere Parteien. Allgemein bedeutet dies: wenn das Risiko eines Sitzverlustes von einer Wahl zur nächsten hoch ist, beeinflusst dies auch die parlamentarische Mitgliederfluktuation. Dabei wird die Wählervolatilität anhand der Nettoveränderungen der Anzahl Sitze im Parlament der Parteien zwischen zwei kantonalen Parlamentswahlen berechnet ${ }^{24}$.

\section{Modell 4: Finanzielle Entschädigungen}

Wie die Studie von Nyffeler et al. ${ }^{25}$ für die kommunale Ebene feststellt, betrachten Mandatsträger das Verhältnis zwischen dem für ein parlamentarisches Amt anfallenden Aufwand und der dafür erhaltenen finanziellen Entschädigung oft als unangemessen. Grundentschädigung, Sitzungsgeld und Spesen, welche die Parlamentarier für ihre Tätigkeit erhalten, werden in den entsprechenden gesetzlichen Erlassen geregelt. Den Befunden von Nyffeler et al. ${ }^{26}$ folgend, kann angenommen werden, dass die Höhe der finanziellen Entschädigung von Mandatsträgern einen positiven Einfluss auf die parlamentarische Mitgliederfluktuationsrate ausübt.

\subsection{Politisch-kulturelle Einflussfaktoren}

\section{Modell 5: politisch-kulturelle Einflussfaktoren}

\section{Sprachregion}

Es liegt auf der Hand, dass die parlamentarische Mitgliederfluktuation nicht ausschliesslich durch institutionelle Anreize und Schranken beeinflusst wird, sondern auch durch politischkulturelle Werte und Einstellungen. Im Folgenden wird unter politischer Kultur in Anlehnung

\footnotetext{
${ }^{21}$ Vgl. Reuven Y. Hazan, Candidate Selection, in: Lawrence LeDuc / Richard G. Niemi / Pippa Norris (Hrsg.), Comparing Democracies 2: New Challenges in the Study of Elections and Voting, London 2002, S. 125.

${ }^{22}$ Vgl. Richard E. Matland / Donley T. Studlar, a.a.O. (Fn. 7).

${ }^{23}$ Vgl. Alan Rosenthal, a.a.O. (Fn. 6).

${ }^{24}$ Vgl. Mogens N. Pedersen, The Dynamics of European Party System: Changing Patterns of Electoral Volatility, in: European Journal of Political Research, 7. Jg. (1979), H. 1, S. 1 - 26.

${ }^{25}$ Vgl. Bettina Nyffeler / Annina Jegher / Stefan Spycher, a.a.O. (Fn. 5).

${ }^{26}$ Vgl. Bettina Nyffeler / Annina Jegher / Stefan Spycher, a.a.O. (Fn. 5).
} 
an Almond und Verba ${ }^{27}$ als die über die Mitglieder einer politischen Gemeinschaft ermittelten kollektiven Werte, Überzeugungen und Einstellungen der Bürgerinnen und Bürger gegenüber der Politik und politischen Objekten verstanden. Aufgrund der sprachlichen Segmentierung erfolgt die politisch-kulturelle Prägung in der Schweiz gemäss Linder ${ }^{28}$ primär in den drei Sprachregionen der deutschen, französischen und italienischen Schweiz. Kriesi et al. ${ }^{29}$ betonen dabei vor allem die unterschiedlichen politischen Werthaltungen zwischen der Bevölkerung der lateinischen und der deutschen Schweiz. Freitag ${ }^{30}$ weist in diesem Zusammenhang darauf hin, „dass die drei Sprachregionen mit Blick auf zivilgesellschaftliche und kulturelle Aspekte ihren Nachbarländern Deutschland, Italien und Frankreich bisweilen ähnlicher sind als ihren innerschweizerischen Nachbarn“. Innerhalb der Schweiz mit ihren verschiedenen kulturellen Räumen unterscheiden sich die damit Vorstellungen und Auffassungen hinsichtlich der Demokratiezufriedenheit im Allgemeinen und der Wirksamkeit der parlamentarischen Arbeit im Speziellen. Untersuchungen zeigen dabei, dass in den französisch- und italienischsprachigen Kantonen das repräsentativ-demokratische Modell einen grösseren Stellenwert einnimmt als das direktdemokratische System, welches vor allem in der Deutschschweiz eine hohe Wertschätzung geniesst. ${ }^{31}$ Entsprechend ist zu erwarten, dass der Stellenwert der Parlamente in der lateinischen Sprachregion grösser ist und der Wettbewerb um Parlamentssitze intensiver ausgetragen wird als in der deutschen Schweiz, was sich auch positiv auf den Mitgliederwechsel auswirkt.

\section{Wahlbeteiligung}

Manifester Ausdruck der politischen Kultur ist auch die Wahlbeteiligung. Entsprechend ist davon auszugehen, dass eine höhere Wertschätzung der Legislativarbeit sowie ein intensiverer Wettbewerb um Parlamentssitze einen mobilisierenden Effekt auf die Beteiligung der Wahlberechtigten bei Parlamentswahlen ausüben, was wiederum einen positiven Einfluss auf den parlamentarischen Mitgliederwechsel hat.

\footnotetext{
${ }^{27}$ Vgl. Gabriel Almond / Sidney Verba, The Civic Culture. Political Attitudes and Democracy in Five Nations, Princeton 1963.

${ }^{28}$ Vgl. Wolf Linder / Isabelle Stadelmann-Steffen, Politische Kultur, in: Ulrich Klöti / Peter Knoepfel / Hanspeter Kriesi / Wolf Linder / Yannis Papadopoulos (Hrsg.), Handbuch der Schweizer Politik, Zürich 2002.

${ }^{29}$ Vgl. Hanspeter Kriesi / Boris Wernli / Pascal Sciarini / Matteo Gianni, Le clivage linguistique: Problèmes de comprehension entre les communautés linguistiques en Suisse, Bundesamt für Statistik, Bern 1996.

${ }^{30}$ Vgl. Markus Freitag, Politische Kultur, in: Peter Knoepfel / Yannis Papadopoulos / Pascal Sciarin /, Adrian Vatter / Silja Häusermann (Hrsg.), Handbuch der Schweizer Politik, Zürich 2014, S. 78.

${ }^{31}$ Vgl. Adrian Vatter, Kantonale Demokratien im Vergleich. Entstehungsgründe, Interaktionen und Wirkungen politischer Institutionen in den Schweizer Kantonen, Opladen 2002.; Marc Bühlmann / Adrian Vatter / Oliver Dlabac / Hans-Peter Schaub, Liberale Romandie, radikale Deutschschweiz? Kantonale Demokratien zwischen Repräsentation und Partizipation, in: Swiss Political Science Review, 19. Jg. (2013), H. 2, S. 157 - 188.; Andreas Ladner, Das Alter der Parlamentarierinnen und Parlamentarier in den kantonalen Parlamenten, in: Parlament, 10. Jg. (2007), H. 2/07, S. 14 - 19.
} 


\section{Interesse der Bürger an der Politik}

Das politische Interesse schafft die Grundlage, dass sich der Einzelne mit politischen Sachfragen auseinandersetzt und bei Bedarf politisch aktiv wird. ${ }^{32}$ Entsprechend ist zu erwarten, dass politische Systeme mit einer stark interessierten Wählerschaft eher zu einer hohen parlamentarischen Mitgliederfluktuationsrate neigen, da die durch ihr politisches Interesse mobilisierte Wählerschaft versucht, über die Wahl starken personalpolitischen Einfluss zu nehmen und eine stärkere Kontrolle ihrer Repräsentanten vornimmt.

\section{Untersuchungsdesign und empirische Befunde}

Zur Überprüfung möglicher Erklärungsfaktoren wird als adäquate statistische Methode die Mehrebenenanalyse gewählt ${ }^{33}$. Mit dieser Methode wird sowohl der Einfluss von kontextuellen wie auch von individuellen Einflussgrössen simultan geschätzt. Im Folgenden wird ein lineares hierarchisches Modell mit Random Intercept und Random Slope ${ }^{34}$ verwendet. Um eine Autokorrelation über die Zeit $\mathrm{zu}$ vermeiden, wurde analog zum Vorgehen von Snijders ${ }^{35}$ eine Zeitvariable gebildet und in die Modelle integriert. ${ }^{36}$

In Tabelle 1 sind die Resultate für die linearen hierarchischen Modelle mit Random Intercept und Random Slope für $\mathrm{t}$ (Modelle 1 bis 5$)^{37}$ zur Erklärung der Niveauunterschiede hinsichtlich der parlamentarischen Mitgliederfluktuationsrate auf subnationaler Ebene

\footnotetext{
${ }^{32}$ Vgl. Oscar W. Gabriel, Politische Einstellungen und politische Kultur, in: Oscar W. Gabriel / Sabine Kropp (Hrsg.), Die EU-Staaten im Vergleich: Strukturen, Prozesse, Politikinhalte, Wiesbaden 2008, S. 201.

${ }^{33}$ Vgl. Kelvyn Jones, Multilevel Approaches to Modelling Contextuality: From Nuisance to Substance in the Analysis of Voting Behaviour, in: Gert P. Westert / René N. Verhoeff (Hrsg.), Places and people: Multilevel modeling in geographical research, Utrecht 1997.; Marco R. Steenbergen / Bradford S. Jones, Modeling Multilevel Data Structures, in: American Journal of Political Science, 46. Jg. (2002), H. 1, S. 218 - 237.

${ }^{34}$ Vgl. Tom Snijders / Roel Bosker, Multilevel Analysis. An introduction to basic and advanced multilevel modeling, London 2012.; Sophia Rabe-Hesketh / Anders Skrondal, Multilevel and Longitudinal Modeling Using Stata: Continuous Responses, College Station 2012.

${ }^{35}$ Vgl. Tom Snijders / Roel Bosker, a.a.O. (Fn. 38).

${ }^{36}$ Indem der zusätzlichen Zeitvariable erlaubt wird, nicht nur zwischen den Kantonen (Intercepts), sondern auch hinsichtlich der Steigung (Slope) zu variieren, wird das ursprüngliche Random Intecept Modell zu einem linearen hierarchischen Random Intercept und Random Slope Modell für die Zeit (t) weiter entwickelt. Ein Vergleich der Random Intercept Modelle mit den komplexeren lineraren hierarchischen Random Intercept und Random Slope Modellen für t über das "Akaike information criterion" (AIC) und das "Bayesian information criterion" (BIC) erlaubt es ferner zu beurteilen, welches der Modelle zur Erklärung der vorliegenden Datenstruktur besser geeignet ist. Da sowohl AIC als auch BIC bei den linearen hierarchischen Random Intercept und Random Slope Modellen für t tiefere Werte ausweisen, kann diesen Modellen eine grössere Erklärungkraft hinsichtlich des Einflusses der Kovariaten auf die parlamentarische Mitgliederflukutationsrate auf subnationaler Ebene als den Random Intercept Modellen zugeschrieben werden, weshalb nachfolgend eben diese komplexeren Random Intercept und Random Slope Modelle für t gerechnet werden.

${ }^{37}$ Die präsentierten Modelle wurden in Stata mit der Empirical Bayes Schätzmethode gerechnet, welche im Gegensatz zu der üblichen Maximum Likelihood Schätzung geringere Schätzfehler prognostiziert. Dieses Vorgehen ermöglicht es darüber hinaus Intervalle zu bilden, innerhalb derer 95\% der Intercepts und Slopes der Kantone erwartet werden (bei Annahme einer Normalverteilung). Diese repräsentieren damit das Spektrum, innerhalb dessen sich die untersuchten Kovariaten mit einer Gewissheit von 95\% vergegenwärtigen.
} 
abgebildet. ${ }^{38}$ Als sozioökonomische und demographische Kontrollgrössen werden analog zu anderen Untersuchungen das durchschnittliche Volkseinkommen und der Urbanisierungsgrad beigezogen.

${ }^{38}$ Auf die Abbildung des Nullmodells wurde verzichtet. 


\begin{tabular}{llllll}
\hline \hline & Modell 1 & Modell 2 & Modell 3 & Modell 4 & Modell 5 \\
\hline Wahlsystem & $3.310^{* * *}$ & & & \\
& $(0.941)$ & & & \\
& & & & \\
Amtszeitbeschränkung & $6.730^{*}$ & & & \\
& $(3.297)$ & & &
\end{tabular}

Parl. Untersuchungskomm.

ständige Kommissionen

0.290

(0.246)

Parlamentsdienst

$1.320 *$

(0.582)

Wählervolatilität

$0.499 * * *$

(0.105)

Parlamentsverkleinerung

$-202.300 * * *$

(20.160)

finanzielle Entschädigung

$0.323+$

(zentriert)

(0.183)

Wahlbeteiligung

(zentriert)

$0.185^{* * *}$

Interesse an Politik

(zentriert)

(0.047)

Sprachregion

$26.700 * *$

(9.661)

$9.441^{* * *}$

(2.573)

Urbanisierung

$11.330^{* *} \quad 11.260 *$

$14.100^{* *}$

$14.430 * *$

15.760 ***

(3.856)

(4.630)

(4.459)

(4.525)

(3.334)

Volkseinkommen

$-0.003$

$-0.045$

$-0.046$

$-0.028$

$-0.059$

(zentriert)

(0.064)

(0.066)

(0.059)

(0.066)

(0.066)

Zeit $t$

0.360 ** $\quad 0.305^{* *}$

$0.350 * * *$

$0.367^{* *}$

$0.566^{* * *}$

(0.112)

(0.114)

(0.102)

(0.116)

(0.116)

\begin{tabular}{llllll} 
Konstante & $19.270^{* * *}$ & $24.260^{* * *}$ & $22.500^{* * *}$ & $25.630^{* * *}$ & $16.290^{* * *}$ \\
& $(4.099)$ & $(4.455)$ & $(4.146)$ & $(4.536)$ & $(3.799)$ \\
\hline Varianz $t$ & $0.022^{* * *}$ & $0.022^{* * *}$ & $0.024^{* * *}$ & $0.025^{* * *}$ & $0.027^{* * *}$ \\
(random slope) & $(0.015)$ & $(0.015)$ & $(0.013)$ & $(0.017)$ & $(0.016)$
\end{tabular}




\begin{tabular}{|c|c|c|c|c|c|}
\hline $\begin{array}{l}\text { Varianz zw. Kantonen } \\
\text { (random intercept Level 2) }\end{array}$ & $\begin{array}{l}31.350 * * * \\
(15.710)\end{array}$ & $\begin{array}{l}52.920 * * * \\
(22.530)\end{array}$ & $\begin{array}{l}46.420 * * * \\
(18.340)\end{array}$ & $\begin{array}{l}46.210^{* * *} \\
(20.490)\end{array}$ & $\begin{array}{l}15.130 * * * \\
(12.030)\end{array}$ \\
\hline Kovarianz $t$ Kantone & $\begin{array}{l}-0.380 \\
(0.425)\end{array}$ & $\begin{array}{l}-0.520 \\
(0.487)\end{array}$ & $\begin{array}{l}-0.295 \\
(0.380)\end{array}$ & $\begin{array}{l}-0.352 \\
(0.476)\end{array}$ & $\begin{array}{l}-0.188 \\
(0.389)\end{array}$ \\
\hline $\begin{array}{l}\text { Varianz zw. Zeitpunkten } \\
\text { (random intercept Level 1) }\end{array}$ & $\begin{array}{l}86.210^{* * *} \\
(6.870)\end{array}$ & $\begin{array}{l}84.040 * * * \\
(6.726)\end{array}$ & $\begin{array}{l}62.450^{* * *} \\
(4.987)\end{array}$ & $\begin{array}{l}85.320 * * * \\
(6.809)\end{array}$ & $\begin{array}{l}84.010^{* * *} \\
(6.702)\end{array}$ \\
\hline Anzahl Kantone & 26 & 26 & 26 & 26 & 26 \\
\hline Anzahl Zeitpunkte & 362 & 362 & 362 & 362 & 362 \\
\hline AIC & 2716 & 2720 & 2624 & 2723 & 2706 \\
\hline
\end{tabular}

Standardfehler in Klammern

$+\mathrm{p}<0.10,{ }^{*} \mathrm{p}<0.05, * * \mathrm{p}<0.01, * * * \mathrm{p}<0.001$

\subsection{Befunde zu den Modellen}

\subsubsection{Befunde zu den einzelnen Modellen}

In Modell 1 werden die Variablen zu den Wahlregeln in das lineare hierarchische Random Intecept und Random Slope Modell ${ }^{39}$ integriert. Dabei zeigt sich, dass sowohl das Wahlsystem als auch eine gesetzlich vorgesehene Amtszeitbeschränkung einen signifikant positiven Einfluss auf die parlamentarische Mitgliederfluktuationsrate ausüben. Bei einem proportional ausgestalteten Wahlsystem fällt der durchschnittliche parlamentarische Mitgliederwechsel signifikant höher aus als in einem Majorzwahlsystem (bzw. Mischsystem). In Modell 1 wie in allen weiteren Modellen zeigt sich zudem, dass die parlamentarische Fluktuationsrate bei einem ansteigenden Urbanisierungsgrad zunimmt, während die Höhe des Volkseinkommens keinen signifikanten Einfluss ausübt. Des Weiteren kann festgehalten werden, dass es im Verlaufe der Untersuchungsperiode zu einem Anstieg der parlamentarischen Mitgliederfluktuationsrate zwischen 0.07\% und 0.64\% kommt. Daraus folgt, dass die Varianz zwischen den Kantonen deutlich grösser ist als diejenige über die Zeit $(t)$, also zwischen den Wahljahren (Beobachtungszeitpunkten). ${ }^{40}$

Modell 2 zur Relevanz des institutionellen Handlungsrahmens kommt im Vergleich zu den anderen Modellen nahezu die geringste Erklärungskraft zu. Immerhin üben die gesetzlich vorgesehene Einsetzung einer parlamentarischen Untersuchungskommission (PUK) zur Kontrolle der Regierungstätigkeit sowie ein gegenüber der übrigen Verwaltung unabhängiger Parlamentsdienst einen signifikant positiven Einfluss auf die Fluktuationsrate aus.

\footnotetext{
${ }^{39}$ Weil die Variablen nicht um den Mittelwert zentriert sind, ist die Interpretation der Konstanten nicht sinnvoll, da diese die Fluktuationsrate eines durchschnittlichen Kantons abbildet, für den Fall, dass alle unabhängigen Variablen den Wert 0 einnehmen. Deswegen wird in der Folge auf eine Interpretation der Konstanten verzichtet.

${ }^{40}$ Dieser Befund gilt für alle getesteten Modelle und wird deswegen in den Erläuterungen zu den nachfolgenden Modellen nicht mehr speziell erwähnt.
} 
Möglicherweise lässt sich dieser Befund auf einen grösseren zeitlichen Arbeitsaufwand zurückführen, welcher durch die Einsetzung einer PUK für die Parlamentarier entsteht. Darüber hinaus wäre auch denkbar, dass die Notwendigkeit der Einsetzung einer PUK zur Kontrolle der Regierungstätigkeit das Vertrauen der Amtsträger in das politische System geschwächt hat und es deshalb zu einem gehäuften Ausscheiden aus den kantonalen Legislativen kommt. Die Anzahl ständiger Kommissionen übt dagegen keinen signifikanten Einfluss auf die parlamentarische Fluktuationsrate aus.

Das Modell 3 erweist sich als besonders erklärungskräftig. So übt eine Parlamentsverkleinerung einen hoch signifikanten Einfluss auf die parlamentarische Mitgliederfluktuationsrate aus, d.h. wird eine kantonale Legislative reduziert, fällt die parlamentarische Mitgliederfluktuationsrate markant höher aus als in Kantonen mit stabilen Legislativen, was auch mit einem erhöhten politischen Wettbewerb begründet werden kann. Im Weiteren zeigt sich, dass die Wählervolatilität einen positiven Einfluss auf die parlamentarische Wechselrate ausübt.

Modell 4 überprüft den Effekt der Vergütungen auf die parlamentarische Mitgliederfluktuation. Dabei wird deutlich, dass die Nettoentschädigung einen moderat signifikanten positiven Einfluss auf den parlamentarischen Mitgliederwechsel ausübt. Mit anderen Worten: Je höher die Vergütung, umso häufiger ist die Fluktuation unter den Abgeordneten. Dieser Befund widerspricht den bisherigen Ergebnissen von Nyffeler ${ }^{41}$ und Kaeslin $^{42}$ auf kommunaler Ebene in der Schweiz. Die Höhe der angesetzten finanziellen Anreize scheint Mandatsträger also entgegen der gängigen Annahme nicht zum Verbleib in einer kantonalen Legislative zu motivieren. Neben der intrinsischen Motivation von Mandatsträgern könnte dafür auch die Freude am freiwilligen Engagement verantwortlich sein. So spielen zwar uneigennützige und wohltätige Aspekte, um sich für das Gemeinwohl einzusetzen, eine zentrale Rolle bei der Übernahme einer ehrenamtlichen Tätigkeit, doch stellt gemäss Stadelmann ${ }^{43}$ für viele der Spass an der Tätigkeit selbst der Hauptgrund ihres freiwilligen Engagements dar. Alternativ liesse sich aber auch argumentieren, dass durch die gestiegene Attraktivität des Mandats aufgrund höherer Entschädigung auch der politische Wettbewerb unter den Kandidierenden gesteigert wird und deshalb die Fluktuation höher ist.

\footnotetext{
${ }^{41}$ Vgl. Bettina Nyffeler / Annina Jegher / Stefan Spycher, a.a.O. (Fn. 5).

${ }^{42}$ Vgl. Isabel Käslin / Raphael Anklin / Madleina Balmer / Tobias Graber, a.a.O. (Fn. 5).

${ }^{43}$ Vgl. Isabelle Stadelmann-Steffen / Richard Traunmüller / Birte Gundelach / Markus Freitag, FreiwilligenMonitor Schweiz 2010, Zürich 2010, S. 17.
} 
Beim Modell 5 zu den Effekten politisch-kultureller Einflussfaktoren wird deutlich, dass alle Variablen einen hoch signifikanten positiven Einfluss auf die parlamentarische Mitgliederfluktuationsrate ausüben: Je höher die Wahlbeteiligung ist, desto deutlicher fällt auch die parlamentarische Mitgliederfluktuation aus. Einen ebenfalls positiven Einfluss übt das Interesse der Bürger an der Politik auf den Parlamentarierwechsel aus. Schliesslich liegt die parlamentarische Fluktuationsrate in mehrheitlich lateinischsprachigen Kantonen auf einem deutlich höheren Niveau als den deutschsprachigen Kantonen.

\subsubsection{Die Befunde zum Gesamtmodell}

Tabelle 2 liefert die Resultate für das Gesamtmodell. Dieses berücksichtigt alle zuvor getesteten Kovariaten, die sich in den jeweiligen Modellen auf einem Niveau von 5\% signifikant erwiesen haben. Mit diesem zweistufigen Vorgehen soll die Robustheit der zuvor erhaltenen Befunde überprüft werden. Die Resultate für das Gesamtmodell zeigen, dass sich die Befunde der Teilmodelle grundsätzlich bestätigen. Es handelt sich um das beste Modell zur Erklärung der Niveauunterschiede zwischen den Kantonen hinsichtlich der parlamentarischen Mitgliederfluktuationsrate. Alle darin berücksichtigten Kovariaten vermögen die Varianz zwischen den Kantonen hinsichtlich der parlamentarischen Mitgliederfluktuationsrate mit einer mehr als 95 prozentiger Sicherheit zu begründen. Im Weiteren ändert sich für keine der ins Modell integrierten Variablen die Einflussrichtung. Demgegenüber stehen allerdings unterschiedlich grosse Veränderungen hinsichtlich ihrer Einflussstärke: Das Wahlsystem verliert deutlich an Erklärungskraft, während kantonale Niveauunterschiede hinsichtlich der parlamentarischen Mitgliederfluktuationsrate durch eine gesetzlich vorgesehene Amtszeitbeschränkung nun deutlich stärker erklärt wird. Alle übrigen Variablen bleiben gegenüber den Teilmodellen auf demselben Niveau signifikant. Entsprechend fallen auch die Veränderungen der Einflussstärke geringfügiger aus. 
Tabelle 2: Gesamtmodell mit allen Kovariaten (5\% Signifikanzniveau)

\begin{tabular}{|c|c|}
\hline & "Gesamtmodell 5\% \\
\hline \multirow[t]{2}{*}{ Wahlsystem } & 1.896* \\
\hline & $(0.748)$ \\
\hline \multirow[t]{2}{*}{ Amtszeitbeschränkung } & $7.755^{* * *}$ \\
\hline & $(2.261)$ \\
\hline \multirow[t]{2}{*}{ Parlamentsdienst } & $0.949 *$ \\
\hline & $(0.457)$ \\
\hline \multirow[t]{2}{*}{ Wählervolatilität } & $0.450 * * *$ \\
\hline & $(0.100)$ \\
\hline \multirow[t]{2}{*}{ Parlamentsverkleinerung } & $-199.900 * * *$ \\
\hline & $(19.770)$ \\
\hline \multirow{2}{*}{$\begin{array}{l}\text { Wahlbeteiligung } \\
\text { (zentriert) }\end{array}$} & $0.154 * * *$ \\
\hline & $(0.042)$ \\
\hline \multirow{2}{*}{$\begin{array}{l}\text { Interesse an Politik } \\
\text { (zentriert) }\end{array}$} & $27.570 * * *$ \\
\hline & $(8.172)$ \\
\hline \multirow[t]{2}{*}{ Sprachregion } & $8.679 * * *$ \\
\hline & $(2.023)$ \\
\hline \multirow[t]{2}{*}{ Urbanisierungsgrad } & $9.574 * * *$ \\
\hline & $(2.609)$ \\
\hline \multirow[t]{2}{*}{ Zeit $t$} & $0.299 * * *$ \\
\hline & $(0.057)$ \\
\hline \multirow[t]{2}{*}{ Konstante } & $17.030 * * *$ \\
\hline & (2.009) \\
\hline \multirow{2}{*}{$\begin{array}{l}\text { Varianz } t \\
\text { (random slope) }\end{array}$} & $0.030 * * *$ \\
\hline & $(0.015)$ \\
\hline \multirow{2}{*}{$\begin{array}{l}\text { Varianz zw. Kantonen } \\
\text { (random intercept Level 2) }\end{array}$} & $14.750 * * *$ \\
\hline & $(10.180)$ \\
\hline \multirow[t]{2}{*}{ Kovarianz $t$ Kantone } & -0.459 \\
\hline & $(0.347)$ \\
\hline \multirow{2}{*}{$\begin{array}{l}\text { Varianz zw. Zeitpunkten } \\
\text { (random intercept Level 1) }\end{array}$} & $59.970 * * *$ \\
\hline & $(4.817)$ \\
\hline Anzahl Kantone & 26 \\
\hline Anzahl Zeitpunkte & 362 \\
\hline$A I C$ & 2592 \\
\hline
\end{tabular}

Standardfehler in Klammern

$\left.\left.\mathrm{p}<0.10,{ }^{*} \mathrm{p}<0.05\right),{ }^{* *} \mathrm{p}<0.01\right),{ }^{* * *} \mathrm{p}<0.001$ 


\section{Fazit: Sprachkultur, Parlamentsverkleinerungen und Amtszeitbeschränkungen als}

\section{Schlüsselfaktoren der parlamentarischen Fluktuation in den Schweizer Kantonen}

Sind es vor allem Wahl- und Parlamentsregeln oder die übergeordnete politische Kultur, die ausschlaggebend sind für das Ausmass an personellen Wechsel in Parlamenten? Diese Frage wurde mittels einer Mehrebenenanalyse für alle 26 kantonalen Legislativen aus verschiedenen Sprachräumen der Schweiz über den Zeitraum von 1960 bis 2012 zu erklären versucht.

Die Befunde machen dabei deutlich, dass die im Zentrum stehende Fragestellung differenziert beantwortet werden muss. So spielen sowohl institutionelle Rahmenbedingungen als auch kulturelle Merkmale eine bedeutende Rolle zur Erklärung der kantonalen Unterschiede der Parlamentsfluktuation. Allerdings sind bei den institutionellen Faktoren nur einige von hoher Relevanz, während andere kaum einen Einfluss ausüben. So erweist sich zunächst die in der Forschung bisher kaum untersuchte Verkleinerung einer Legislative als insgesamt stärkster Einflussfaktor aller untersuchten Grössen. Aufgrund des längeren Untersuchungszeitraums im Vergleich zu früheren Studien ${ }^{44}$ kommt damit die Bedeutung dieser längerfristig wirkenden Massnahme voll zum Tragen und bestätigt auch die wenigen internationalen Befunde. ${ }^{45}$ Die Reduktion der Parlamentsmandate führt in rund der Hälfte der Kantone, die diese Massnahme beschlossen haben, zu einer markant höheren parlamentarischen Mitgliederfluktuation als in denjenigen Kantonen, die auf diese Regeländerung verzichtet haben. Signifikant in der theoretisch erwarteten Richtung, wenn auch auf einem geringeren Niveau erweisen sich zudem die Ausgestaltung des Wahlsystems sowie eine gesetzlich vorgesehene Amtszeitbeschränkung. In Kantonen mit Proporzwahlsystem und einer Amtszeitbefristung (in der Regel von zwölf Jahren) findet ein signifikant häufigerer Austausch der politischen Repräsentanten statt als in denjenigen mit Majorz- oder Mischwahlsystemen ohne Amtszeitbeschränkung. Nur eine geringe Rolle spielen hingegen Faktoren, die die Stärke und den Handlungsspielraum einer Legislative gegenüber der Exekutive erfassen. So vermag etwa die Anzahl ständiger Parlamentskommissionen die kantonalen Niveauunterschiede hinsichtlich der parlamentarischen Mitgliederfluktuation nicht zu erklären. Die Ursache für die geringe Relevanz ist dabei im gesteigerten Arbeitsaufwand für die nur Teilzeit arbeitenden Parlamentarier zu vermuten, die mit einer steigenden Zahl an Kommissionsmitgliedschaften verbunden ist. Überraschenderweise eine geringere, fast zu vernachlässigende Rolle kommt den für ein parlamentarisches Amt ausbezahlten finanziellen Vergütungen zu. Dieser Befund lässt sich am ehesten mit der individuellen Motivationslage der Mandatsträger an ihrer

\footnotetext{
${ }^{44}$ Vgl. Till Heinsohn / Markus Freitag, a.a.O. (Fn. 2).

${ }^{45}$ Vgl. Gary F. Moncrief / Richard G. Niemi / Lynda W. Powell, a.a.O. (Fn.19).
} 
Nebentätigkeit begründen, bei der das öffentliche Prestige, die Möglichkeiten der politischen Einflussnahme und die intrinsische Befriedigung an der parlamentarischen Arbeit im Vordergrund steht. ${ }^{46}$

Welche Rolle spielen neben den institutionellen Spielregeln politisch-kulturelle Aspekte wie die Zugehörigkeit zu einem bestimmten Sprachraum oder die Wertschätzung des Parlaments und das politische Interesse der Bevölkerung? Die Befunde sind eindeutig. Alle politischkulturellen Grössen erweisen sich als äusserst bedeutsam zur Erklärung des Mitgliederwechsels in kantonalen Parlamenten und machen deutlich, dass neben den institutionellen Anreizsystemen auch die politischen Einstellungen, Meinungen und Überzeugungen der Wählenden im Aggregat einen beträchtlichen Einfluss auf den Umgang mit politischen Ämtern ausüben. In der französischsprachigen Schweiz mit einem höheren Stellenwert und grösseren Vertrauen in repräsentativ-demokratische Organe ist der Wettbewerb um parlamentarische Mandate ausgeprägter, was schliesslich auch zu einer stärkeren Fluktuation der Abgeordneten als in der Deutschschweiz führt.

Damit zeigt sich insgesamt ein differenziertes Bild in Bezug auf die Relevanz institutioneller und kultureller Faktoren zur Erklärung des parlamentarischen Mitgliederwechsels. Während Massnahmen wie die Senkung der Anzahl Parlamentsmandate und die Amtszeitbeschränkung einen direkten und starken Einfluss auf die Fluktuationsrate ausüben, erweisen sich die Höhe der finanziellen Vergütungen und institutionelle Regeln zur Stärkung des Parlaments gegenüber der Exekutive im vorliegenden Fall ohne nachhaltigen Einfluss. Gleichzeitig erweisen sich langfristige politisch-kulturelle Merkmale für das im Zentrum stehende Phänomen als mindestens ebenso wichtig wie einzelne institutionelle Arrangements. Als relativ unbedeutend erweisen sich schliesslich sozioökonomische und demographische Faktoren mit Ausnahme des Urbanisierungsgrads zur Erklärung der kantonalen Niveauunterschiede der parlamentarischen Mitgliederfluktuation.

Im Kontext einer steigenden Belastung der parlamentarischen Entscheidungsträger in einer zunehmend komplexen Umwelt stellt sich damit abschliessend die Frage, ob populäre Sparentscheide wie die Verkleinerung von Parlamenten oder die Vergrösserung von Wahlkreisen nicht längerfristig einer funktionierenden Demokratie abträglich sind. So führt offenbar die Reduktion der Legislativmandate nicht nur zu einer höheren Arbeitsbelastung für die einzelnen Mandatsträger, sondern am Ende auch zu einem signifikant höheren parlamentarischen Mitgliederwechsel, worunter ohne Zweifel die Qualität der Gesetzgebungsund Aufsichtstätigkeit der Legislative leidet.

\footnotetext{
${ }^{46}$ Vgl. Isabelle Stadelmann-Steffen / Richard Traunmüller / Birte Gundelach / Markus Freitag, a.a.O. (Fn. 47).
} 


\begin{abstract}
Der Beitrag liefert für die Schweiz eine Analyse der parlamentarischen Mitgliederfluktuation auf subnationaler Ebene für alle 26 kantonalen Legislativen für den Zeitraum zwischen 1960 und 2012. Im Mittelpunkt steht die Frage, ob es institutionelle Regeln oder Merkmale der politischen Kultur sind, die den Wechsel in Parlamenten erklären. Die Befunde machen dabei deutlich, dass sowohl institutionelle Rahmenbedingungen als auch kulturelle Merkmale eine bedeutende Rolle zur Erklärung der kantonalen Unterschiede der Parlamentsfluktuation. Während Massnahmen wie die Senkung der Anzahl Parlamentsmandate und die Amtszeitbeschränkung einen direkten und starken Einfluss auf die Fluktuationsrate ausüben, erweisen sich die Höhe der finanziellen Vergütungen und institutionelle Regeln zur Stärkung des Parlaments gegenüber der Exekutive ohne nachhaltigen Einfluss. Gleichzeitig stellen sich langfristige politisch-kulturelle Merkmale wie die Zugehörigkeit zu einem Sprachraum für das im Zentrum stehende Phänomen als mindestens ebenso wichtig heraus wie einzelne institutionelle Arrangements. Als relativ unbedeutend erweisen sich schliesslich sozioökonomische und demographische Faktoren mit Ausnahme des Urbanisierungsgrads zur Erklärung der kantonalen Niveauunterschiede der parlamentarischen Mitgliederfluktuation.
\end{abstract}

